\title{
ON THE STRUCTURE OF THE GRADE THREE PERFECT IDEALS OF TYPE THREE
}

\author{
Eun Jeong Choi, Oh-Jin Kang, and Hyoung June Ko
}

\begin{abstract}
Buchsbaum and Eisenbud showed that every Gorenstein ideal of grade 3 is generated by the submaximal order pfaffians of an alternating matrix. In this paper, we describe a method for constructing a class of type 3 , grade 3 , perfect ideals which are not Gorenstein. We also prove that they are algebraically linked to an even type grade 3 almost complete intersection.
\end{abstract}

\section{Introduction}

An ideal $I$ in a Noetherian local ring $R$ with maximal ideal $\mathfrak{m}$ is perfect if the length of a maximal $R$-sequence contained in $I$, the grade of $I$, is the same as the projective dimension of $R / I$. When $I$ is a perfect ideal of grade $g$, the type of $I$, denoted by type $I$, is the dimension of $R / \mathfrak{m}$-vector space $\operatorname{Ext}_{R}^{g}(R / \mathfrak{m}, R / I)$. Equivalently, if

$$
\mathbb{F}: 0 \longrightarrow F_{g} \longrightarrow F_{g-1} \longrightarrow \cdots \longrightarrow F_{1} \longrightarrow R
$$

is the minimal free resolution of $R / I$, then type $I=\operatorname{rank} F_{g}$. An ideal $I$ of grade $g$ is a complete intersection if $I$ can be generated by a regular sequence $x_{1}, x_{2}, \ldots, x_{g}$. It is well-known that such an ideal is a perfect ideal of type 1. More generally, a perfect ideal is said to be Gorenstein if it has type 1. A perfect ideal of grade $g$ is an almost complete intersection if it can be minimally generated by $g+1$ elements.

For long years, many people have been studying the structure of classes of perfect ideals. Burch [4] proved a structure theorem for grade 2 perfect ideals. The Hilbert-Burch theorem asserts that every perfect ideal of grade 2 is generated by maximal minors of a certain matrix. Buchsbaum and Eisenbud [3] proved a structure theorem for Gorenstein ideals of grade 3 which says that every Gorenstein ideal of grade 3 is generated by the submaximal order pfaffians of a certain alternating matrix. They also showed a structure theorem for almost complete intersections of grade 3 . The self duality and commutativity

Received March 11, 2008; Revised July 5, 2008.

2000 Mathematics Subject Classification. 13C05, 13C14,13C40, 14M10.

Key words and phrases. perfect ideal of grade 3, skew-symmetrizable matrix, minimal free resolution. 
of the algebra structure on the minimal free resolutions of $R / I$, where $I$ is a Gorenstein ideal, was exploited to understand the structure of the perfect ideal I. Brown [2] gave a structure theorem for a class of type 2, grade 3 perfect ideals and $\lambda(I)=\operatorname{dim}_{k} \Lambda_{1}^{2}>0$, where $\lambda(I)$ is the numerical invariant. The number $\lambda(I)$ was used by Kustin and Miller [8] to distinguish classes of grade 4 Gorenstein ideals $I$ in terms of free resolutions of $R / I$. Sanchez [10] proved a structure theorem for a class of type 3 , grade 3 perfect ideals and $\lambda(I) \geq 2$. In [6] we introduced a generalized alternating matrix to be a skew-symmetrizable matrix and gave a structure theorem for grade 4 complete intersection ideals. A generalized alternating matrix has been further elaborated to describe the structure theorem for a class of type 2 , grade 3 perfect ideals $I$ minimally generated by an odd number $n \geq 5$ elements [7]. The main purpose of this paper is to describe and produce a new class of type 3 , grade 3 perfect ideals which is not Gorenstein.

In Section 2, we introduce useful properties of pfaffians, and review the theory of linkage. In Section 3, we give a new class of non-Gorenstein type 3, grade 3 perfect ideals minimally generated by the quotients of the submaximal order pfaffians of the alternating matrix induced by a skew-symmetrizable matrix. We also show that the ideals in this class are geometrically linked to an even type, grade 3 almost complete intersection.

\section{Preliminaries}

To describe the structure theorems mentioned in the introduction, we need some properties of an alternating matrix. An alternating matrix is a square skew-symmetric matrix whose diagonal entries are zero. Let $T=\left(t_{i j}\right)$ be an $n \times n$ alternating matrix with entries in a commutative ring $R$. Then it turns out that the determinant of an alternating matrix $T$ is a square of a homogeneous polynomial of degree $\frac{n}{2}$ in $R$ and is zero when $n$ is odd. The pfaffian of $T$ is defined as the uniquely determined square root of the determinant of $T$ and is denoted by $\operatorname{Pf}(T)$. We define $\operatorname{Pf}_{s}(T)$ to be an ideal generated by the $s$ th order pfaffians of $T$. If $s<n$, we let $T\left(i_{1}, i_{2}, \ldots, i_{s}\right)$ denote the alternating submatrix of $T$ obtained by deleting rows and columns $i_{1}, i_{2}, \ldots, i_{s}$ from $T$. Let $(i)=i_{1}, i_{2}, \ldots, i_{s}$ denote the index of integers. Let $\theta(i)$ denote the sign of permutation that rearranges $(i)$ in increasing order. If $(i)$ has a repeated index, then we set $\theta(i)=0$. Let $\tau(i)$ be the sum of the entries of $(i)$. Define

$$
T_{(i)}=(-1)^{\tau(i)+1} \theta(i) \operatorname{Pf}\left(T\left(i_{1}, i_{2}, \ldots, i_{s}\right)\right) .
$$

If $s=n$, we let $T_{(i)}=(-1)^{\tau(i)+1} \theta(i)$ and if $s>n$, we let $T_{(i)}=0$. Let $\mathbf{t}=\left[\begin{array}{llll}T_{1} & T_{2} & \cdots & T_{n}\end{array}\right]$ be the row vector of the pfaffians of $T$ of order $n-1$ signed appropriately according to the conventions described above. Pfaffians can be developed along a row just like the determinants. There is a "Laplace expansion" for developing pfaffians in terms of ones of lower order. 
Lemma $2.1([8])$. Let $T$ be an $n \times n$ alternating matrix and $j$ a fixed integer, $1 \leq j \leq n$. Then

(1) $\operatorname{Pf}(T)=\sum_{i=1}^{n} t_{i j} T_{i j}$, and
(2) $\mathbf{t} T=0$.

The following lemma follows from Lemma 2.1 .

Lemma $2.2([10])$. Let $T$ be an $n \times n$ alternating matrix. Let $a, b, c, d$, and $e$ be distinct integers between 1 and $n$. Then

(1) $\sum_{i=1}^{n} t_{i k} T_{i a b}=-\delta_{k a} T_{b}+\delta_{k b} T_{a}$

(2) $\sum_{i=1}^{n} t_{i k} T_{i a b c}=\delta_{k a} T_{b c}-\delta_{k b} T_{a c}+\delta_{k c} T_{a b}$,

(3) $\sum_{i=1}^{n} t_{i k} T_{i a b c d}=-\delta_{k a} T_{b c d}+\delta_{k b} T_{a c d}-\delta_{k c} T_{a b d}+\delta_{k d} T_{a b c}$

(4) $\sum_{i=1}^{n} t_{i k} T_{i a b c d e}=\delta_{k a} T_{b c d e}-\delta_{k b} T_{a c d e}+\delta_{k c} T_{a b d e}-\delta_{k d} T_{a b c e}+\delta_{k e} T_{a b c d}$,

where $\delta_{i j}$ is the Kronecker's delta.

The following lemma which is a direct consequence from Lemma 2.1 and Lemma 2.2 will be used in the sequel.

Lemma 2.3. Let $n$ be a positive integer and $T$ an $n \times n$ alternating matrix. Assume that $i, j, k$, and $l$ are elements of a set $\{1,2, \ldots, n\}$. Then

$$
T_{i} T_{j k l}+T_{j} T_{i k l}+T_{k} T_{i j l}+T_{l} T_{i j k}=0 .
$$

The Buchsbaum-Eisenbud structure theorem identifies every grade 3 Gorenstein ideal as the ideal $\mathrm{Pf}_{n-1}(T)=\left(T_{1}, T_{2}, \ldots, T_{n}\right)$ of a certain $n \times n$ alternating matrix $T$.

Theorem 2.4 ([3]). Let $R$ be a Noetherian local ring with maximal ideal $\mathfrak{m}$.

(1) Let $n \geqslant 3$ be an odd integer. Let $F$ be a free $R$-module with rank $F=n$. Let $f: F^{*} \rightarrow F$ be an alternating map whose image is contained in $\mathfrak{m} F$. Suppose that $\mathrm{Pf}_{n-1}(f)$ has grade 3 . Then $\mathrm{Pf}_{n-1}(f)$ is a Gorenstein ideal minimally generated by $n$ elements.

(2) Every grade 3 Gorenstein ideal arises as in this way.

We notice that as in [3] or [9], in most cases, linkage is used in the case of perfect ideals in Gorenstein or Cohen-Macaulay local rings. However the result that we use here is true for perfect ideals in any commutative ring, as shown by Golod [5].

Definition 2.5. Let $I$ and $J$ be perfect ideals of grade $g$. An ideal $I$ is linked to $J, I \sim J$ if there exists a regular sequence $\mathbf{x}=x_{1}, x_{2}, \ldots, x_{g} \in I \cap J$ such 
that $J=(\mathbf{x}): I$ and $I=(\mathbf{x}): J$, and geometrically linked to $J$ if $I \sim J$ and $I \cap J=(\mathbf{x})$.

A fundamental result is that linkage is a symmetric relation on the set of perfect ideals in a Noetherian ring $R$.

Theorem 2.6 ([9]). Let $R$ be a Noetherian ring. If I is a grade $g$ perfect ideal and $\mathbf{x}=x_{1}, x_{2}, \ldots, x_{g}$ is a regular sequence in $I$, then $J=(\mathbf{x}): I$ is a grade $g$ perfect ideal and $I=(\mathbf{x}): J$.

An almost complete intersection of grade $g$ is linked to a grade $g$ Gorenstein ideal by a regular sequence $\mathbf{x}$.

Proposition $2.7([3])$. Let $I$ and $J$ be perfect ideals of the same grade $g$ in a Noetherian local ring $R$ and suppose that $I$ is linked to $J$ by a regular sequence $\mathbf{x}=x_{1}, x_{2}, \ldots, x_{g}$. Then

(1) If I is Gorenstein, then $J=(\mathbf{x}, w)$ for some $w \in R$.

(2) If $J$ is minimally generated by $\mathbf{x}$ and $w$, then $I$ is Gorenstein.

Now we review the structure theorems for a class of $\lambda(I)>0$, type 2, grade 3 perfect ideals $I$ and for a class of $\lambda(I) \geq 2$, type 3 , grade 3 perfect ideals $I$ given by Brown [2] and Sanchez [10], respectively.

Let $I$ be any ideal in a Noetherian local ring $R$. Let $(\mathbb{F}, d)$ be a minimal free resolution of $R / I$. Let $C$ be the image of $d_{2}$ and $K$ the submodule of $C$ which is generated by the Koszul relations on the entries of $d_{1}$. We note that if $I$ is minimally generated by $r_{1}, r_{2}, \ldots, r_{n}$, and $\left\{e_{1}, e_{2}, \ldots, e_{n}\right\}$ is a basis of $F_{1}$, then $K$ is generated by the set $\left\{r_{j} e_{i}-r_{i} e_{j} \mid 1 \leq i<j \leq n\right\}$. Define

$$
\lambda(I)=\operatorname{dim}_{k}(K+\mathfrak{m} C) / \mathfrak{m} C,
$$

where $\mathfrak{m}$ is the maximal ideal of $R$ and $k=R / \mathfrak{m}$. Since $\lambda(I)$ is the maximum number of minimal generators of $K$ which can be chosen to be the part of a minimal basis for $C$, we see that $\lambda(I)$ is also the maximum number of Koszul relations which can appear as rows of a matrix for $d_{2}$. Brown gave a structure theorem for a class of $\lambda(I)>0$, type 2 , grade 3 perfect ideals $I$. The minimal free resolution $\mathbb{F}$ of $R / I$ is described in [2].

Theorem 2.8 ([2]). Let $R$ be a Noetherian local ring with maximal ideal $\mathfrak{m}$. Let $n>4$ be an integer. Let $I$ be a type 2, grade 3 perfect ideal minimally generated by $n$ elements. If $\lambda(I)>0$, then there is an $n \times n$ alternating matrix $T=\left(t_{i j}\right)$ with $t_{12}=0$ and $t_{i j} \in \mathfrak{m}$ such that

(1) if $n$ is odd, then $I=\left(T_{1}, T_{2}, z_{1} T_{12 j}+z_{2} T_{j}: 3 \leq j \leq n\right)$ for some $z_{1}, z_{2} \in \mathfrak{m}$,

(2) if $n$ is even, then $I=\left(\operatorname{Pf}(T), T_{12}, z_{1} T_{1 j}+z_{2} T_{2 j}: 3 \leq j \leq n\right)$ for some $z_{1}, z_{2} \in \mathfrak{m}$.

Sanchez gave a structure theorem for a class of $\lambda(I) \geq 2$, type 3 , grade 3 perfect ideals $I$. The minimal free resolution $\mathbb{F}$ of $R / I$ is described in [10]. 
Theorem 2.9 ([10]). Let $R$ be a Noetherian local ring with maximal ideal $\mathfrak{m}$. Let $I$ be a type 3 , grade 3 perfect ideal minimally generated by $n>4$ elements. If $\lambda(I) \geq 2$, then there exists an $n \times n$ alternating matrix $T=\left(t_{i j}\right)$ and a $2 \times 3$ matrix $X=\left(x_{i j}\right)$ with $t_{i j}, x_{i j} \in \mathfrak{m}$ such that

(1) If $n>3$ is odd, then either

$$
\begin{aligned}
I= & \left(T_{1}, x_{11} T_{2}+x_{12} T_{3}+x_{13} T_{123}, x_{21} T_{2}+x_{22} T_{3}+x_{23} T_{123},\right. \\
& \left.\Delta_{3} T_{j}+\Delta_{2} T_{12 j}+\Delta_{1} T_{13 j} \mid 4 \leq j \leq n\right)
\end{aligned}
$$

or

$$
\begin{aligned}
I= & \left(T_{123}, x_{11} T_{1}+x_{12} T_{2}+x_{13} T_{3}, x_{21} T_{1}+x_{22} T_{2}+x_{23} T_{3},\right. \\
& \left.\Delta_{3} T_{12 j}+\Delta_{2} T_{13 j}+\Delta_{1} T_{23 j} \mid 4 \leq j \leq n\right),
\end{aligned}
$$

where $\Delta_{i}$ is the determinant of the $2 \times 2$ submatrix of $X$ obtained by deleting the ith column.

(2) If $n>3$ is even, then either

$$
\begin{aligned}
I= & \left(\operatorname{Pf}(T), x_{11} T_{12}+x_{12} T_{13}+x_{13} T_{23}, x_{21} T_{12}+x_{22} T_{13}+x_{23} T_{23},\right. \\
& \left.\Delta_{3} T_{1 j}+\Delta_{2} T_{2 j}+\Delta_{1} T_{123 j} \mid 4 \leq j \leq n\right)
\end{aligned}
$$

or

$$
\begin{aligned}
I= & \left(T_{12}, x_{11} \operatorname{Pf}(T)+x_{12} T_{13}+x_{13} T_{23}, x_{21} \operatorname{Pf}(T)+x_{22} T_{13}+x_{23} T_{23},\right. \\
& \left.\Delta_{3} T_{1 j}+\Delta_{2} T_{2 j}+\Delta_{1} T_{123 j} \mid 4 \leq j \leq n\right) .
\end{aligned}
$$

\section{Perfect ideals of grade 3}

In this section we construct a new class of non-Gorenstein type 3 , grade 3 perfect ideals generated by certain quotients of the submaximal order pfaffians of the alternating matrix induced by some skew-symmetrizable matrix.

To this end, we begin this section with the definition of a generalized alternating matrix.

Definition 3.1. Let $R$ be a commutative ring with identity. An $n \times n$ matrix $X$ over $R$ is said to be generalized alternating or skew-symmetrizable if there exist nonzero diagonal matrices $D^{\prime}=\operatorname{diag}\left\{u_{1}, u_{2}, \ldots, u_{n}\right\}$ and $D=$ $\operatorname{diag}\left\{w_{1}, w_{2}, \ldots, w_{n}\right\}$ with entries in $R$ such that $D^{\prime} X D$ is an alternating matrix.

We denote by $G A_{n}$ the set of all $n \times n$ skew-symmetrizable matrices over $R$. Let $X$ be an $n \times n$ skew-symmetrizable matrix. We denote by $\mathcal{A}(X)$ the alternating matrix induced by $X$ as follows:

$$
\mathcal{A}(X)= \begin{cases}X & \text { if } X \text { is alternating, } \\ D^{\prime} X D & \text { if } X \text { is not alternating. }\end{cases}
$$

The following lemma gives us relations between the determinants of the skew-symmetrizable matrices and the pfaffians of the alternating matrices induced by the skew-symmetrizable matrices. 
Lemma 3.2. Let $X$ be an $n \times n$ skew-symmetrizable matrix and $\mathcal{A}(X)$ the alternating matrix $D^{\prime} X D$ induced by $X$ defined in (3.1). Let $\hat{u}_{i}=\prod_{j=1}^{n} u_{j} / u_{i}$ and $\hat{w}_{i}=\prod_{j=1}^{n} w_{j} / w_{i}$ for $i=1,2, \ldots, n$. Let $X(i)$ be the $(n-1) \times(n-1)$ submatrix of $X$ obtained by deleting the ith row and the corresponding column of it. Then

$$
\hat{u}_{i} \hat{w}_{i} \operatorname{det} X(i)=\mathcal{A}(X)_{i}^{2} .
$$

Proof. It follows from the basic properties of determinants of square matrices and pfaffians of alternating matrices.

Example 3.3. Let $R$ be a Noetherian local ring with maximal ideal $\mathfrak{m}$ and let $n$ be an odd integer with $n>3$. Let $Y=\left(y_{i j}\right)$ be an $n \times n$ alternating matrix with $y_{12}=0$ and entries in $\mathfrak{m}$ and $A$ the submatrix of $Y$ obtained by deleting the first two columns and the last $(n-2)$ rows of $Y$ from $Y$. For two elements $v, w \in \mathfrak{m}$, we define the $n \times n$ skew-symmetrizable matrix $G_{1}$ by

$$
G_{1}=\left[\begin{array}{c|c}
B & v A \\
\hline-A^{t} & Y(1,2)
\end{array}\right], \quad \text { where } B=\left[\begin{array}{cc}
0 & w \\
-w & 0
\end{array}\right],
$$

and $Y(1,2)$ is the $(n-2) \times(n-2)$ alternating submatrix of $Y$ obtained by removing the first, second rows and columns from $Y$. The alternating matrix $\mathcal{A}\left(G_{1}\right)$ is obtained by multiplying the first two columns of $G_{1}$ by $v$.

Let $x_{i}$ be an element defined by

$$
x_{i}=\mathcal{A}\left(G_{1}\right)_{i} / v \text { for } i=1,2,3, \ldots, n .
$$

We define $\overline{\mathrm{Pf}_{n-1}\left(G_{1}\right)}$ to be the ideal generated by $n$ elements $x_{i}$. The next theorem says that $\overline{\mathrm{Pf}_{n-1}\left(G_{1}\right)}$ characterizes a perfect ideal $I$ of grade 3 satisfying the following properties : (1) $I$ has type 2, (2) the number of generators for $I$ is odd, and (3) $\lambda(I)>0$.

Theorem 3.4 ([7]). Let $R$ be a Noetherian local ring with maximal ideal $\mathfrak{m}$. Let $n$ be an odd integer with $n>3$ and $v, w$ elements in $\mathfrak{m}$. Let $G_{1}$ be the $n \times n$ skew-symmetrizable matrix defined in (3.2). Then

(1) if $I=\overline{\mathrm{Pf}_{n-1}\left(G_{1}\right)}$ is an ideal of grade 3 with $\lambda(I)>0$, then $I$ is a perfect ideal of type 2 .

(2) Every perfect ideal of grade 3, type 2, $\lambda(I)>0$ minimally generated by $n$ elements arises as in the way of (1).

Proof. See the proof of Theorem 4.3 [7].

Now we construct a skew-symmetrizable matrix which defines a type 3 , grade 3 perfect ideal. Let $A=\left(a_{i j}\right)$ and $Y=\left(y_{i j}\right)$ be an $r \times 3$ matrix and an $r \times r$ alternating matrix with entries in $\mathfrak{m}$, respectively. Set $F$ to be the $3 \times r$ matrix 
defined by

$$
F=\left[\begin{array}{cccc}
a_{11} & a_{21} & \cdots & a_{r 1} \\
-a_{12} & -a_{22} & \cdots & -a_{r 2} \\
a_{13} & a_{23} & \cdots & a_{r 3}
\end{array}\right]
$$

with an even integer $r \geq 4$. Define an $(r+3) \times(r+3)$ skew-symmetrizable matrix $G_{2}$ by

$$
G_{2}=\left[\begin{array}{c|c}
\mathbf{0} & \bar{F} \\
\hline F^{t} & Y
\end{array}\right], \text { where } \bar{F}=\left[\begin{array}{cccc}
v a_{11} & v a_{21} & \cdots & v a_{r 1} \\
-u a_{12} & -u a_{22} & \cdots & -u a_{r 2} \\
u v a_{13} & u v a_{23} & \cdots & u v a_{r 3}
\end{array}\right]
$$

with $u, v \in \mathfrak{m}$. The alternating matrix $\mathcal{A}\left(G_{2}\right)$ is obtained by multiplying the first column of $G_{2}$ by $v$, the second column of it by $u$, and the third column of it by $u v$. To describe non-Gorenstein perfect ideal of grade 3, we need the ideal $\overline{\mathrm{Pf}_{r+2}\left(G_{2}\right)}$ induced from the submaximal order pfaffians of $\mathcal{A}\left(G_{2}\right)$ as follows.

Definition 3.5. With notations as above, we let

$$
\mathcal{A}\left(G_{2}\right)=\left[\begin{array}{c|c}
\mathbf{0} & \bar{F} \\
\hline-\bar{F}^{t} & Y
\end{array}\right]
$$

be the alternating matrix induced by $G_{2}$. We define $\overline{\mathrm{Pf}_{r+2}\left(G_{2}\right)}$ to be the ideal generated by the quotients of the submaximal order pfaffians of $\mathcal{A}\left(G_{2}\right)$ by $u v$,

$$
\overline{\mathrm{Pf}_{r+2}\left(G_{2}\right)}=\left(\mathcal{A}\left(G_{2}\right)_{1} / u v, \mathcal{A}\left(G_{2}\right)_{2} / u v, \ldots, \mathcal{A}\left(G_{2}\right)_{r+3} / u v\right) .
$$

Next, we prove our main theorem which gives a class of type 3 , grade 3 perfect ideals algebraically linked to a type $r$, grade 3 almost complete intersection.

Theorem 3.6. Let $R$ be a Noetherian local ring with maximal ideal $\mathfrak{m}$. With notations as above, let $x_{1}=\mathcal{A}\left(G_{2}\right)_{1} / u v, x_{2}=\mathcal{A}\left(G_{2}\right)_{2} / u v, x_{3}=\mathcal{A}\left(G_{2}\right)_{3} / u v$. If $\mathbf{x}=x_{1}, x_{2}, x_{3}$ is a regular sequence in $\overline{\mathrm{Pf}_{r+2}\left(G_{2}\right)}$, then $(1)(\mathbf{x}): \overline{\mathrm{Pf}_{r+2}\left(G_{2}\right)}$ is a type even $r$, grade 3 almost complete intersection and $(2) \overline{\mathrm{Pf}_{r+2}\left(G_{2}\right)}$ is a type 3 , grade 3 perfect ideal.

Proof. (1) Define an $(r+3) \times(r+3)$ alternating matrix $T$ by

$$
T=\widetilde{G}_{2}=\left[\begin{array}{c|c}
\mathbf{0} & F \\
\hline-F^{t} & Y
\end{array}\right] .
$$

Then we observe that

$$
\begin{aligned}
& x_{1}=u T_{1}, x_{2}=v T_{2}, x_{3}=T_{3}, x_{k}=\mathcal{A}\left(G_{2}\right)_{k} / u v=u v T_{k} \\
& \quad \text { for } k=4,5, \ldots, r+3 .
\end{aligned}
$$


To see that $\mathrm{Pf}_{r+2}(T)$ is a Gorenstein ideal under the hypothesis of the theorem, we first note that by Lemma $2.3, \overline{\mathrm{Pf}_{r+2}\left(G_{2}\right)}$ has grade 3 . Next, since $\overline{\mathrm{Pf}_{r+2}\left(G_{2}\right)} \subset \mathrm{Pf}_{r+2}(T), \mathrm{Pf}_{r+2}(T)$ has grade 3 by Lemma 2.3. Finally, since $T$ is an alternating matrix, $\operatorname{Pf}_{r+2}(T)$ is a Gorenstein ideal by Theorem 2.4. Let $\mathbf{y}=y_{1}, y_{2}, y_{3}$ be a sequence of elements in $R$, where $y_{1}=T_{1}, y_{2}=T_{2}, y_{3}=T_{3}$. Since $\mathbf{x}$ is a regular sequence, $\mathbf{y}$ is also regular sequence. By Theorem 2.6 and Proposition 2.7, $J=(\mathbf{y}): \mathrm{Pf}_{r+2}(T)$ is a grade 3 perfect ideal, and $J$ is an almost complete intersection. We claim that $J$ has type even $r$ and $J=(\mathbf{x}): \overline{\mathrm{Pf}_{r+2}\left(G_{2}\right)}$. Direct computation gives us

$$
\operatorname{Pf}(Y) T_{k} \in(\mathbf{y}) \text { for } k=4,5,6, \ldots, r+3 \text {. }
$$

Hence we have

$$
J=(\mathbf{y}, \operatorname{Pf}(Y)) .
$$

Since $u$ and $v$ are contained in $\mathfrak{m}$, the fact that $J$ has type $r$ follows from the Bass result ([1] Proposition 2.9), which says that if $J$ is a grade 3 perfect ideal algebraically linked to a perfect ideal $\mathrm{Pf}_{r+2}(T)$ by a regular sequence $\mathbf{y}$ in $J$, then the type of $J$ is equal to the minimal number of generators for the canonical module $\operatorname{Ext}_{R}^{3}(R / J, R)$ and

$$
\operatorname{Ext}_{R}^{3}(R / J, R) \cong(\mathbf{y}): J /(\mathbf{y}) \cong \operatorname{Pf}_{r+2}(T) /(\mathbf{y})
$$

From (3.4) and (3.5), it is easy to see that

$$
J=(\mathbf{x}): \overline{\mathrm{Pf}_{r+2}\left(G_{2}\right)} .
$$

(2) We will prove that $\overline{\mathrm{Pf}_{r+2}\left(G_{2}\right)}$ is a type 3 , grade 3 perfect ideal. Since we have already shown that $\overline{\mathrm{Pf}_{r+2}\left(G_{2}\right)}$ is a grade 3 perfect ideal, it suffices to prove that the type of $\overline{\mathrm{Pf}_{r+2}\left(G_{2}\right)}$ is 3 . From the Bass result and

$$
\operatorname{Ext}_{R}^{3}\left(R / \overline{\mathrm{Pf}_{r+2}\left(G_{2}\right)}, R\right) \cong(\mathbf{x}): \overline{\mathrm{Pf}_{r+2}\left(G_{2}\right)} /(\mathbf{x}) \cong J /(\mathbf{x}),
$$

we have type $\overline{\mathrm{Pf}_{r+2}\left(G_{2}\right)}=3$.

The minimal free resolution $\mathbb{F}$ of $R / \overline{\mathrm{Pf}_{r+2}\left(G_{2}\right)}$ is given by

$$
\mathbb{F}: 0 \longrightarrow R^{3} \stackrel{f_{3}}{\longrightarrow} R^{r+5} \stackrel{f_{2}}{\longrightarrow} R^{r+3} \stackrel{f_{1}}{\longrightarrow} R
$$

where

$$
f_{1}=\left[\begin{array}{llll}
x_{1} & x_{2} & x_{3} \cdots & x_{r+3}
\end{array}\right], f_{2}=\left[\begin{array}{ccc}
\mathbf{0} & \bar{F} & B \\
-F^{t} & Y & \mathbf{0}
\end{array}\right], f_{3}=\left[\begin{array}{c}
C \\
Q \\
N
\end{array}\right]
$$


and

$$
\begin{aligned}
B & =\left[\begin{array}{cc}
0 & x_{3} \\
x_{3} & 0 \\
-x_{2} & -x_{1}
\end{array}\right], C=\left[\begin{array}{ccc}
0 & -\operatorname{Pf}(Y) & T_{1} \\
\operatorname{Pf}(Y) & 0 & T_{2} \\
0 & 0 & T_{3}
\end{array}\right], \\
Q & =\left[\begin{array}{ccc}
-q_{21} & q_{11} & T_{4} \\
-q_{22} & q_{12} & T_{5} \\
\vdots & \vdots & \vdots \\
-q_{2 r} & q_{1 r} & T_{r+3}
\end{array}\right], \quad N=\left[\begin{array}{lll}
0 & u & 0 \\
v & 0 & 0
\end{array}\right], \\
q_{i j} & =(-1)^{i+1} \sum_{1 \leq k \leq r} Y_{j k} a_{k i} \text { for } i=1,2 .
\end{aligned}
$$

The following example illustrates Theorem 3.6.

Example 3.7. Let $R=\mathbb{C}[[x, y, z]]$ be the formal power series ring over the field $\mathbb{C}$ of the complex numbers with indeterminates $x, y, z$. Let $A$ and $Y$ be a $4 \times 3$ matrix and a $4 \times 4$ alternating matrix, respectively, given by

$$
A=\left[\begin{array}{ccc}
0 & z & -y \\
-y & z & 0 \\
z & -x & 0 \\
0 & 0 & -x
\end{array}\right] \text { and } Y=\left[\begin{array}{cccc}
0 & x & 0 & z \\
-x & 0 & 0 & 0 \\
0 & 0 & 0 & y \\
-z & 0 & -y & 0
\end{array}\right]
$$

Then $F$ is the $3 \times 4$ matrix given by

$$
F=\left[\begin{array}{cccc}
0 & -y & z & 0 \\
-z & -z & x & 0 \\
-y & 0 & 0 & -x
\end{array}\right]
$$

We set $u=x$ and $v=y$ and define $G_{2}$ to be a $7 \times 7$ skew-symmetrizable matrix given by

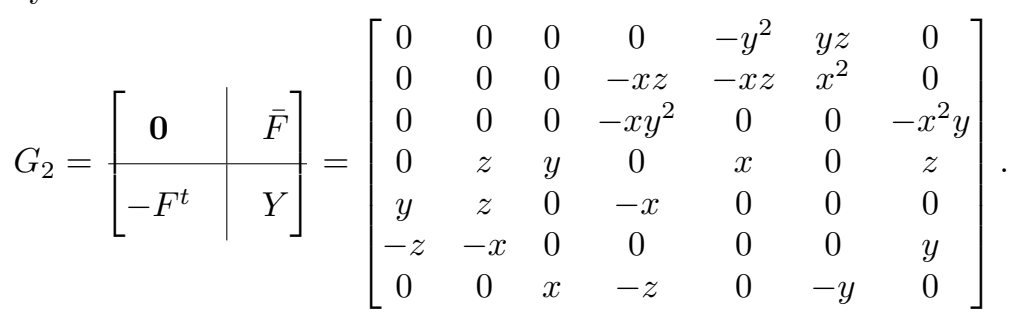

Define the $7 \times 7$ alternating matrix $T=\widetilde{G}_{2}$ by

$$
T=\widetilde{G}_{2}=\left[\begin{array}{c|c}
\mathbf{0} & F \\
\hline-F^{t} & Y
\end{array}\right] .
$$

Then we will show that $\overline{\mathrm{Pf}_{6}\left(G_{2}\right)}=\left(x^{4}+x y^{2} z,-y^{4}-x^{2} y z, x y z+y^{2} z-\right.$ $\left.z^{3}, x^{3} y^{2}-x^{2} y z^{2}, x^{2} y z^{2}, x^{2} y^{2} z,-x^{2} y^{3}+x y^{2} z^{2}\right)$ is a type 3 , grade 3 perfect ideal and linked to a type 4 , grade 3 almost complete intersection by a regular 
sequence $\mathbf{x}=x^{4}+x y^{2} z,-y^{4}-x^{2} y z,-z^{3}+x y z+y^{2} z$. The minimal free resolution $\mathbb{F}$ of $R / \overline{\mathrm{Pf}_{6}\left(G_{2}\right)}$ has the form:

$$
\mathbb{F}: 0 \longrightarrow R^{3} \stackrel{f_{3}}{\longrightarrow} R^{9} \stackrel{f_{2}}{\longrightarrow} R^{7} \stackrel{f_{1}}{\longrightarrow} R,
$$

where the map $f_{2}$ has the following matrix:

$$
f_{2}=\left[\begin{array}{ccccccccc}
0 & 0 & 0 & 0 & -y^{2} & y z & 0 & 0 & x_{3} \\
0 & 0 & 0 & -x z & -x z & x^{2} & 0 & x_{3} & 0 \\
0 & 0 & 0 & -x y^{2} & 0 & 0 & -x^{2} y & -x_{2} & -x_{1} \\
0 & z & y & 0 & x & 0 & z & 0 & 0 \\
y & z & 0 & -x & 0 & 0 & 0 & 0 & 0 \\
-z & -x & 0 & 0 & 0 & 0 & y & 0 & 0 \\
0 & 0 & x & -z & 0 & -y & 0 & 0 & 0
\end{array}\right]
$$

To describe $f_{1}$ and $f_{3}$ in concise form, we set

$$
\begin{aligned}
& x_{1}=x^{4}+x y^{2} z, \quad x_{2}=-y^{4}-x^{2} y z, \quad x_{3}=x y z+y^{2} z-z^{3}, \\
& x_{4}=x^{3} y^{2}-x^{2} y z^{2}, \quad x_{5}=x^{2} y z^{2}, \quad x_{6}=x^{2} y^{2} z, \quad x_{7}=-x^{2} y^{3}+x y^{2} z^{2}, \\
& T_{1}=x^{3}+y^{2} z, T_{2}=-y^{3}-x^{2} z, T_{3}=x y z+y^{2} z-z^{3}, T_{4}=x^{2} y-x z^{2}, \\
& T_{5}=x z^{2}, \quad T_{6}=x y z, T_{7}=-x y^{2}+y z^{2} .
\end{aligned}
$$

Then we have

$$
\begin{aligned}
f_{1} & =\left[\begin{array}{lllllllll}
x_{1} & x_{2} & x_{3} & x_{4} & x_{5} & x_{6} & x_{7}
\end{array}\right], \\
f_{3} & =\left[\begin{array}{ccccccccc}
0 & -x y & 0 & -y z & x z+y z & z^{2} & -x^{2} & 0 & y \\
x y & 0 & 0 & y^{2} & -z^{2} & -y z & x z & x & 0 \\
T_{1} & T_{2} & T_{3} & T_{4} & T_{5} & T_{6} & T_{7} & 0 & 0
\end{array}\right]^{t} .
\end{aligned}
$$

If $K$ is the ideal generated by $x_{1}, x_{2}, x_{3}$, then the radical of $K$ is equal to the maximal ideal $\mathfrak{m}=(x, y, z)$. Since $\mathfrak{m}$ has grade $3, \mathbf{x}=x_{1}, x_{2}, x_{3}$ is a regular sequence and so $\overline{\mathrm{Pf}_{6}\left(G_{2}\right)}$ has grade 3 by Lemma 2.3. Now we will show that $(\mathbf{x}): \overline{\mathrm{Pf}_{6}\left(G_{2}\right)}$ is a type 4 , grade 3 almost complete intersection. Let $y_{1}=$ $T_{1}, y_{2}=T_{2}, y_{3}=T_{3}$. Since $\mathbf{x}$ is a regular sequence, $\mathbf{y}=y_{1}, y_{2}, y_{3}$ is a regular sequence. By Theorem 2.4 and Proposition $2.7, I=\left(T_{1}, T_{2}, \ldots, T_{7}\right)$ is a grade 3 Gorenstein ideal, and $J=(\mathbf{y}): I$ is a grade 3 almost complete intersection. Since $w=\operatorname{Pf}(Y)=-x y, J=(\mathbf{y}, w)=\left(-x^{3}-y^{2} z,-y^{3}-x^{2} z,-y^{2} z+z^{3},-x y\right)$. As seen in the proof of Theorem 3.6, we have $J=(\mathbf{x}): \overline{\operatorname{Pf}_{6}\left(G_{2}\right)}$. From the Bass result and

$$
\begin{aligned}
\operatorname{Ext}_{R}^{3}(R / J, R) & \cong(\mathbf{x}): J /(\mathbf{x}) \cong \overline{\operatorname{Pf}_{6}\left(G_{2}\right)} /(\mathbf{x}) \\
& =\left(\mathbf{x}, x y T_{4}, x y T_{5}, x y T_{6}, x y T_{7}\right) /(\mathbf{x})
\end{aligned}
$$

we have type $J=4$. By the same method, $\overline{\mathrm{Pf}_{6}\left(G_{2}\right)}$ has type 3 . 


\section{References}

[1] H. Bass, On the ubiquity of Gorenstein rings, Math. Z. 82 (1963), 8-28.

[2] A. Brown, A structure theorem for a class of grade three perfect ideals, J. Algebra 105 (1987), 308-327.

[3] D. A. Buchsbaum and D. Eisenbud, Algebra structures for finite free resolutions and some structure theorems for ideals of codimension 3, Amer. J. Math. 99 (1977), no. 3, 447-485.

[4] L. Burch, On ideals of finite homological dimension in local rings, Proc. Cambridge Philos. Soc 64 (1968), 941-948.

[5] E. S. Golod, A note on perfect ideals, from the collection "Algebra" (A. I. Kostrikin, $E d)$, Moscow State Univ. Publishing House (1980), 37-39.

[6] O.-J. Kang and H. J. Ko, The structure theorem for Complete Intersections of grade 4, Algebra. Collo. 12 (2005), no. 2, 181-197.

[7] - Structure theorem for perfect ideals of grade g, Comm. Korean. Math. Soc. 21 (2006), no 4, 613-630.

[8] A. Kustin and M. Miller, Structure theory for a class of grade four Gorenstein ideals, Trans. Amer. Math. Soc. 270 (1982), 287-307.

[9] C. Peskine and L. Szpiro, Liaison des variétés algébriques, Invent. Math. 26 (1974), 271-302.

[10] R. Sanchez, A structure theorem for type 3, grade 3 perfect ideals, J. Algebra 123 (1989), $263-288$.

Eun JEONG CHOI

UNIVERSITY COLLEGE

YONSEI UNIVERSITY

SeOul 120-749, KoreA

E-mail address: eunjchoi@yonsei.ac.kr

OH-JIN KANG

Department of Mathematics

SCience of College

UNIVERSITY OF INCHEON

INCHEON 402-749, KorEA

E-mail address: ohkang@incheon.ac.kr

Hyoung June Ko

DePARTMENT OF MATHematics

Yonsei UNIVERSITY

SEOUl 120-749, KoreA

E-mail address: hjko@yonsei.ac.kr 